\title{
Microvascular rarefaction
}

\section{The decline and fall of blood vessels}

\author{
Michael S. Goligorsky \\ Departments of Medicine, Pharmacology and Physiology; Renal Research Institute; New York Medical College; Valhalla, NY USA
}

Key words: endothelial cell migration, endothelial cell dysfunction, endorepellin, endostatin, endothelial-mesenchymal transition, cell reprogramming

Abbreviations: ECD, endothelial cell dysfunction; eNOS, endothelial nitric oxide synthase; VEGF, vascular endothelial growth factor; TIMP, tissue inhibitor of metalloprotease; Ang-2, angiopoietin-2; DMA, asymmetric dimethylarginine; GFP, green fluorescent protein; FGF, fibroblast growth factor; TGFbeta, transforming growth factor-beta

Submitted: 10/25/09

Accepted: 10/26/09

Previously published online: www.landesbioscience.com/journals/ organogenesis/article/10427

Correspondence to: Michael S. Goligorsky; Email:michael_goligorsky@nymc.edu
$\mathrm{T}$ he goals of this presentation are twofold: (1) to briefly sketch the field of vascular rarefaction as a key component of various fibrotic diseases and (2) to illustrate it with four vignettes depicting diverse mechanisms of microvascular rarefaction. Specifically, I shall describe migratory and angiogenic incompetence of endothelial cells under conditions of reduced bioavailability of nitric oxide, role of endothelial-to-mesenchymal cell and mesenchymal stem cell-to-endothelial reprogramming, and potential role of antiangiogenic peptides in the development of graft vascular disease as exemplified by chronic allograft nephropathy.

\section{Introduction}

Identification of the role played by neovascularization in tumor progression ${ }^{1}$ triggered an avalanche of investigations into the mechanisms of angiogenesis (reviewed in refs. 2-6), to name a few. Four general mechanisms were described as (1) sprouting angiogenesis, (2) intussusceptive microvascular growth, (3) vasculogenesis and (4) looping angiogenesis. ${ }^{7}$ Yet, the majority of chronic fibrotic processes are characterized by the opposite trend, namely the drop-out of blood vessels, which could potentially accelerate the progression of fibrotic tissue degeneration. This latter consideration, combined with the therapeutic requirement to suppress angiogenesis in tumors, served as a major drive to the field of anti-angiogenesis. In this brief overview, I shall dwell mostly on the microvascular rarefaction in chronic diseases: its molecular and cellular mechanisms and sequelae. Specifically, I shall present four vignettes, seemingly unrelated, but all illustrating various aspects of vascular rarefaction with the emphasis on endothelial cell dysfunction (ECD) and angiogenic incompetence. In addition, I'll provide data on endothelial and mesenchymal cell reprogramming, which may contribute to impaired angiogenesis and vasculogenesis.

\section{Historical Note}

Microvascular adaptation has long been in focus of investigations into its role in hypertension and other diseases. ${ }^{8}$ In the skeletal muscle, reduction in arteriole and capillary density was documented in 12-14 week-old (but not in 6-8 week-old) spontaneously hypertensive rats (SHR).? Of interest is the finding that when SHR were placed in a hypobaric chamber simulating high altitude, the abnormalities in blood pressure and vascular density were significantly diminished. ${ }^{10}$ In the cremaster muscle of rats with one-kidney, one-clip hypertension, videomicroscopy revealed an early enhanced vasoconstriction resulting in the closure of the smaller arterioles, followed by the structural rarefaction in the maintenance phase of hypertension. ${ }^{11}$ In rats with coarctation of the abdominal aorta model of hypertension, the density of 3-4-order arterioles was reduced by $19 \%$, despite the normal-to-reduced blood pressure in the femoral artery, invoking a suggestion of existence of some hypertension-independent mechanisms of vascular rarefaction. ${ }^{12}$ Hansen-Smith et al. ${ }^{13}$ described a rapid loss of microvessels in the cremaster muscle of rats subjected to renal mass reduction with high-salt diet (hypertensive), but also in sham-operated rats 
receiving a high-salt diet (normotensive). Return of rats to the low-salt diet resulted in the reversal of hypertension and microvascular rarefaction. ${ }^{14}$ Interestingly, in this model of renal mass reduction and highsalt intake, microvascular rarefaction was not observed in female rats. ${ }^{15}$ Gobe et al. ${ }^{16}$ provided evidence that endothelial cell apoptosis, especially after one week of establishing Goldblatt model of hypertension, was at least in part responsible for development of microvascular rarefaction. This observation was confirmed in a model of congestive heart failure in New Zealand White rabbits which develop microvascular rarefaction in the skeletal muscle. ${ }^{17}$

Microvascular rarefaction was detected in the myocardium of rats with chronic inhibition of nitric oxide synthase, characteristic of the pressureoverload hypertrophy ${ }^{18}$ and in eNOS ${ }^{-1}$ mice. ${ }^{19}$ Diabetes-induced microvascular rarefaction appears to be more severe than that seen in NOS inhibition. ${ }^{20}$ In rats with reduced renal mass, Tornig et al. ${ }^{21}$ described an increase in myocardial arteriolar wall thickness, reduction in capillary density and widening of the interstitium. In rats with angiotensin II-dependent cardiac hypertrophy capillary density was reduced by $18 \%$ whereas the arteriolar density was $54 \%$ increased, and both processes were found to be independently regulated since losartan prevented changes in capillary, but not arteriolar density. ${ }^{22}$

Microvascular rarefaction in different forms of hypertension and in association with aging was described in the brain. ${ }^{23,24}$ Similarly, microvascular rarefaction in the eyes was observed in humans with essential hypertension and in spontaneously hypertensive rats. ${ }^{25,26}$ The most dramatic example of vessel regression is provided by the hyperoxic treatment of premature babies and development of retinopathy of prematurity: in the first phase regression of immature retinal blood vessels is driven by the suppression of VEGF, whereas the subsequent switch to normoxic breathing leads to a hypoxic generation of VEGF and an overshoot angiogenesis. ${ }^{27}$

The density of skin capillaries was found to be decreased in the aging human skin, in preeclampsia, chronic heart failure, in essential hypertension and in chronic renal failure. ${ }^{28-32}$ Using two different approaches, video microscopy and laser Doppler imaging, investigators showed that the functional capillary rarefaction manifests itself in the reduced recruitment of capillaries during postocclusive hyperemia in patients with essential hypertension and chronic renal failure. ${ }^{31,32}$

There is strong evidence that renal microvascular rarefaction is a constant companion of many renal diseases leading to glomerular sclerosis and tubulointerstitial fibrosis. ${ }^{33-40}$ In patients with IgA nephropathy, Namikoshi et al. ${ }^{41}$ showed that early stages of disease were associated with the increased expression of VEGF without reduction in the number of peritubular capillaries, whereas capillary density was decreased in advanced stages despite the maintenance of VEGF expression. Similar observations on reduction of sinusoidal capillaries density were made in rat liver fibrogenesis. ${ }^{42}$

Computer modeling of the effect of microvascular rarefaction on perfusion and resistance (percolation phenomenon) resulted in the approximation that network resistance sustained only minor changes with the reduction of capillary density of less than $30 \%$, but further decrease in capillary density led to a double exponential increase in the resistance. ${ }^{43}$ In a mathematical model of the hamster cheek pouch microcirculation, Greene et al. ${ }^{44}$ calculated the maximal effect on the resistance reaching 21\% for vascular rarefaction and $75 \%$ for vasoconstriction. At the same time, vascular rarefaction resulted in a much more profound heterogeneity of blood flow in various vessel orders.

\section{Molecular Mechanisms of Microvascular Rarefaction: "Drive in Reverse" and Anti-Angiogenic Reprogramming}

Accrued knowledge of processes responsible for embryonic and adult angiogenesis has impacted on the understanding of vascular regression as a drive in reverse. This point of view is certainly pertinent to cases when removal of angiogenic stimuli results in microvascular rarefaction. The typical case is retinopathy of prematurity, when early treatment with high concentrations of oxygen results in obliteration of immature retinal vessels, whereas later, upon resumption of breathing air and cessation of hyperoxic therapy, compensatory angiogenesis with formation of leaky vessels takes place in the retina and vitreous body. ${ }^{45}$ In the first phase of the disease, hyperoxic suppression of VEGF leads to vascular pruning, while the opposite scenario unveils in the normoxic phase, when compensatory overshoot in VEGF synthesis takes place. Interestingly, by alleviating the first phase with placental growth factor acting through VEGFR-1, development of retinopathy can be prevented. This example of abrupt VEGF deficiency and overdrive is, however, atypical for the most pathological conditions where changes in angiogenic and anti-angiogenic factors are much more subtle. A list of conditions associated with impaired angiogenesis, either excessive or insufficient, is steadily growing to incorporate, in addition to cancers, some infectious diseases, Alzheimer disease, atherosclerosis, hypertension and deteriorating functions of transplanted organs, obesity, psoriasis and Kaposi's sarcoma, diabetic retinopathy, asthma, primary pulmonary hypertension, inflammatory bowel, endometriosis, ovarian hyperstimulation syndrome, preeclampsia, arthritis, to name a few. ${ }^{46}$

An alternative to the "drive in reverse" is a view, supported by multitude of studies into pathophysiologic conditions that ascribes microvascular rarefaction to the induction of specialized antiangiogenic programs. The best-known examples are angiostatin and endostatin discovered through a systematic search for antiangiogenic mediators. ${ }^{47}$ Since then, the list of some-such endogenous anti-angiogenic substances has grown impressively to incorporate angiostatin, alphastatin, arrestin, anti-thrombin III, canstatin, interferon-beta, 2-methoxyestradiol, PEDF, platelet factor 4, tetrahydrocortisol, thrombospondin-1, TIMP-2, tumstatin, to name a few, ${ }^{48}$ [see Table 1].

Endothelial cells are capable of generating both pro- and anti-angiogenic substances. Im et al. ${ }^{49}$ demonstrated that inhibition of a lysosomal protease cathep$\sin \mathrm{B}$ in cultured endothelial cells resulted 
in the expression of VEGF mRNA and protein, thus eliminating the requirement for exogenous VEGF in the capillary tube formation assay. In addition, blockade of cathepsin B activity lead to the reduction of endothelial production of endostatin, an anti-angiogenic fragment of collagen XVIII. On the other hand, overexpression of cathepsin B reduced VEGF-dependent capillary tube formation. Another product of endothelial cells, endostatin, interacts with integrins $\alpha 5$ and $\alpha \mathrm{V}$ on endothelial cells. ${ }^{50}$ The resulting physiological effect depends on whether endostatin is immobilized or soluble: in the former case it supports endothelial cell survival and migration, in the latter, it inhibits cell migration.

The duality of the process of vascular remodeling is best encapsulated in the fact of existence of splice variants of VEGF possessing either pro- or anti-angiogenic properties. Traditionally well-studied isoforms of VEGF-A such as VEGF ${ }_{189}$, VEGF $_{165}$ and VEGF $_{121}$ (termed VEGFxxx) are all angiogenic. In contrast, the use of an alternate 3 ' splice acceptor site in the exon 8 results in generation of VEGFxxxb isoforms, which possess anti-angiogenic properties. ${ }^{51} \mathrm{VEGFb}$ isoforms are downregulated in tumors like renal cell carcinoma, colorectal and prostate carcinomas, with their expression inversely correlating with tumor growth rate. However, in diabetics with proliferative retinopathy $\mathrm{VEGFb}$ expression is suppressed, whereas in patients with preeclampsia the coordinated regulation of pro- and anti-angiogenic isoforms is disrupted. ${ }^{51,52}$

Indeed, type 2 diabetes represents an ample example of angiogenic transformation. Using ex vivo fragments of renal cortex and medulla embedded in growth factor-depleted matrigel, we have observed an angiogenic switch during natural history of disease-from pro-angiogenic phenotype at the early, pre-diabetic stages of the metabolic syndrome in 8 weeks-old Zucker diabetic fat rats to the profound inhibition of ex vivo angiogenesis and microvascular rarefaction in the kidneys of 22 week-old animals (Fig. 1), which by this time exhibit severe ECD. ${ }^{40}$ This angiogenic switch emphasizes the importance of staging a disease for a better assessment of the vascular contribution.

Table 1. Factors contributing to microvascular rarefaction

(I) Removal of angiogenic stimuli

(2) Discontinuation of blood flow

(3) Disruption of endothelial pericyte association

(4) Angiogenesis inhibitors:

(a) Upregulation of endogenous TSP-I and TSP-2

(b) Angiopoietin 2

(c) Interferons (via decreased expression of bFGF and VEGF)

(d) Chemokines (PF-4, Mig, Inf-inducible prot-10)

(e) Soluble FIt-I and Tie-2

(f) Cleavage products of matrix proteins (arresten, canstatin, tumstatin, vastatin, restin, endostatin, endorepellin)

(g) Proteinases, enzymes or plasma proteins (PEX from MMP-2, mini-TrpRS from tryptophanyl-tRNA synthetase, angiostatin from plasminogen, $16 \mathrm{~K}$ prolactin from prolactin serpin fragments)

(5) Endothelial dysfunction

(6) Endothelial or stem cell reprogramming:

(a) Endothelial-mesenchymal transition

(b) Mesenchymal-endothelial transition

\section{The Concept of Podokinesis and Its Relevance to Angiogenic Competence}

In a series of studies ${ }^{53-55}$ of cultured endothelial cells, we observed that ostensibly stationary cells are constantly and spontaneously changing tractional forces applied to focal adhesions and, when cultured on a thin silicone film, the wrinkling pattern undergoes perpetual changes. We termed this scalar motion "podokinesis." 55 This scalar motility is NO-dependent and inhibition of NO synthase in endothelial cells reduced its activity and hampered cell migration in the wound-healing assay. When presented with a guidance cues, podokinetic endothelial cells exhibited migratory behavior, whereas cells with inhibited podokinesis were halted in their directed migration. Schematic illustration of podokinesis and its necessity for migration is presented in Figure 2. Given the fact that reduction in bioavailable $\mathrm{NO}$ is a hallmark of ECD, these findings ignited future studies of angiogenesis in various conditions associated with impairment of endothelial functions.

\section{Mechanical Forces in the Process of Regression}

Mechanical forces may play a significant role in angiogenic vis-à-vis anti- angiogenic reprogramming. Cyclic strain triggers endothelial cell programs which result in sprouting angiogenesis and recruitment of smooth muscle cells. ${ }^{56}$ Endothelial secretory paracrine signals under strained conditions were identified as angiopoietin-2 and PDGF. In a recently described wound healinginduced re-routing of peripheral microvasculature toward the granulation tissue by contracting myofibroblasts, ${ }^{57}$ formation of these vascular loops provided the first line of defense. On the other hand, tortuousity of microvessels and stagnation of blood flow may lead to reduction of shear stress-maintained endothelial phenotype with compromised eNOS function, leading initially to prolonged periods of functional closure culminating eventually in structural closure of the lumen.

Morphologic and chronologic patterns of regressing blood vessels, as studied in the rabbit corneas, ${ }^{58}$ appear to be invariant and follow the sequence of events such as endothelial cell damage, platelet adhesion and degranulation, stasis of blood, perivascular monocytic infiltration, degeneration of the vessel wall cells and removal of vascular debris by macrophages. Many of these steps have been confirmed in later studies, as detailed below.

Morphologic patterns of regression of the papillary membrane and hyaloids 


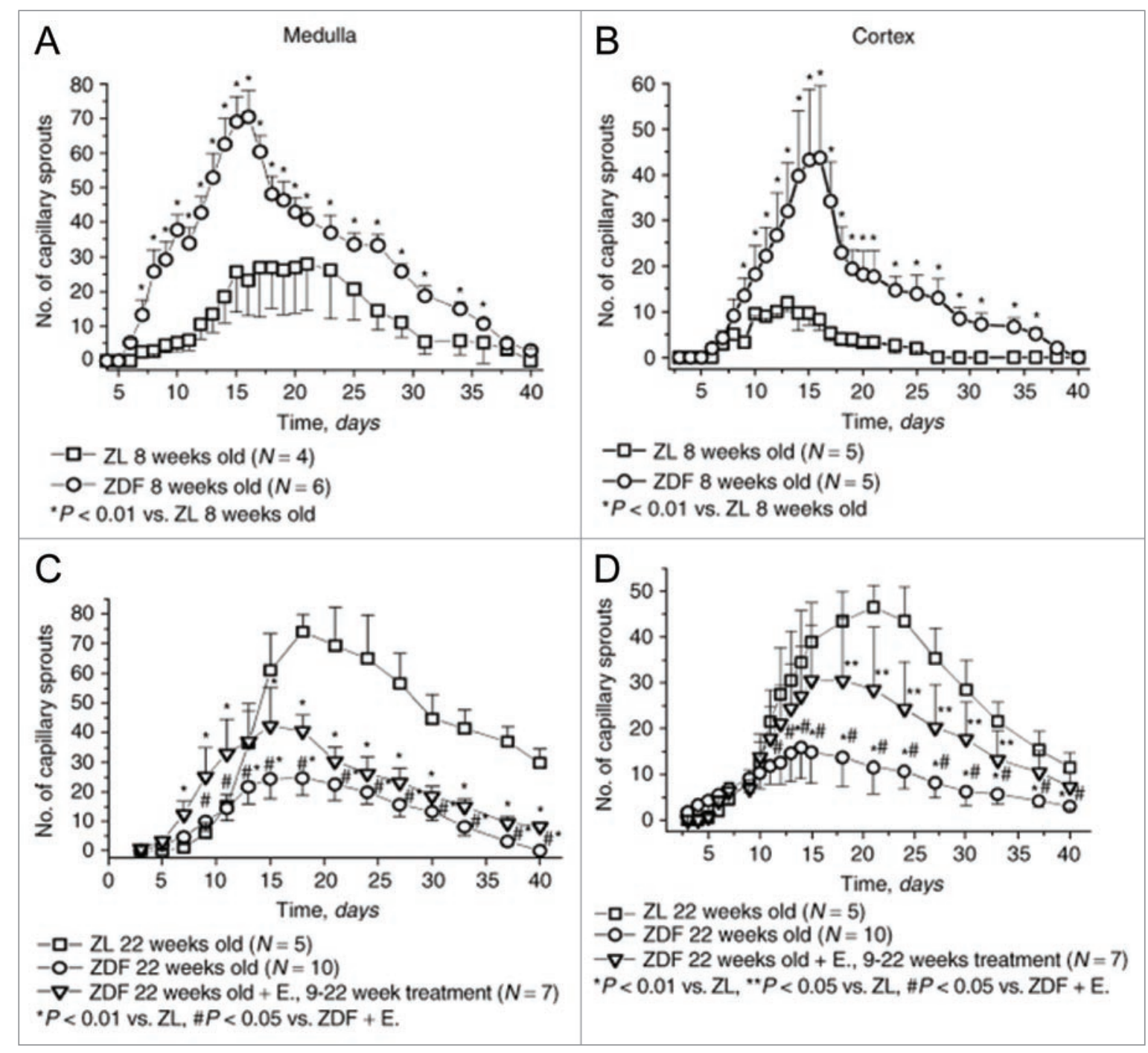

Figure 1. Ex vivo angiogenesis in 3-dimensional matrigel cultures of fragments of the renal cortex or medulla obtained from 8-week-old and 22-weekold Zucker diabetic fat rats. Note that at the time of initiation of diabetes angiogenesis is enhanced, whereas at 22 weeks of age, when ECD is rampant, capillary sprouting is suppressed. These ex vivo findings were supported by the finding of a similar dynamics of microvascular density in the kidneys of these rats. Modified from ref. 40.

vessels were studied in newborn mice. ${ }^{59}$ It was found that regression occurred segmentally and resulted in decreased number of interconnections. Macrophages were abundantly attracted to regressing vasculature, which shows many apoptotic endothelial cells and pericytes. At later times, vessels were obstructed. One of the molecular mechanisms deployed by macrophages consists in the short-range secretion of WNT7b which triggers apoptotic program in endothelial cells. ${ }^{60}$

The regression of blood vessels may be driven in part by platelet aggregation along the dysfunctional endothelium and/ or stagnant flow, release of angiostatin, a cleavage product of plasminogen/plasmin by matrix metalloproteinases (all released from activated platelets) with the ensuing collapse of the capillary bed. ${ }^{61}$ In addition to this scenario, endothelial-mesenchymal transition (EndoMT) may be similarly involved in the decreased patency of blood vessels (vide infra).

The role of pericytes is of critical importance. ${ }^{62}$ On the one hand, these cells stabilize blood vessels and maintain constant communication with endothelial cells. Their recruitment in many blood vessels is governed by PDGF-B (enhancement) and ang-2 (inhibition) secreted by the endothelium, and is dependent on $\mathrm{N}$-cadherin hemophilic interactions, $\alpha 4 \beta 1$ integrins, activity of the membrane type-1 matrix metalloproteinase and O-glycans for vessel stabilization. Deficiency of any of stabilizing factors would lead to the regression of the vessel. On the other hand, enhanced contractile activity of pericytes has profound sequelae for the vascular function. Yemisci et al. ${ }^{63}$ demonstrated that protracted contraction of microvascular pericytes in the postischemic period is responsible for capillary contraction (no-reflow phenomenon) and entrapment of erythrocytes. Oxidonitrosative stress of ischemia-reperfusion deemed to be causatively involved in this phenomenon because administration of an anti-oxidant/peroxynitrite scavenger, ebselen, reversed protracted contraction 
and erythrocyte entrapment. Thus, pericytes may play an important role in the "functional" stage of microvascular occlusion, even before vessels become vestigial.

\section{Reprogramming: Endothelial-Mesenchymal Transition}

It has been appreciated that during embryonic development Flk-1-positive vascular progenitor cells have a potential to differentiate into either endothelial or smooth muscle cells. ${ }^{64}$ DeRuiter et al. ${ }^{65}$ have documented the potential of embryonic endothelial cells to transdifferentiate into mesenchymal cells expressing smooth muscle cell markers. These observations posed a broader question on the potential single progenitor cell type for endothelial and smooth muscle cells and the possibility of conversion of endothelial to smooth muscle cells during adulthood. In vitro studies of cultured bovine and human endothelial cells showed that gamma-irradiation, mitomycin $\mathrm{C}$, or FGF deprivation induce endothelial transdifferentiation into smooth muscle cells. ${ }^{66,67}$ There is indication that similar processes may occur in vivo during neointimal formation in patients with transplant arteriosclerosis- $\alpha-S M \quad$ actin-positive neointimal cells are derived from recipient's circulating endothelial progenitors. ${ }^{68}$ The possibility and the potential role of a similar transdifferentiation process in vascular remodeling during other pathologic processes until recently has not been considered. Significant literature exists on the role of epithelial-mesenchymal transdifferentiation in development and progression of fibrosis. ${ }^{69-72}$ Yet, the role of endothelial-mesenchymal transdifferentiation in vascular regression and fibrosis remains unexplored.

We have screened the effect of eNOS inhibition on the expression of "cardiovascular-relevant" genes in human umbilical vein endothelial cells and generated a model of non-hypertensive nephrosclerosis using $\mathrm{NO}$ inhibitors at subpressor doses, which showed regression of renal microvasculature, as previously detailed. ${ }^{73}$ The finding of increased endothelial synthesis of collagen XVIII and excessive

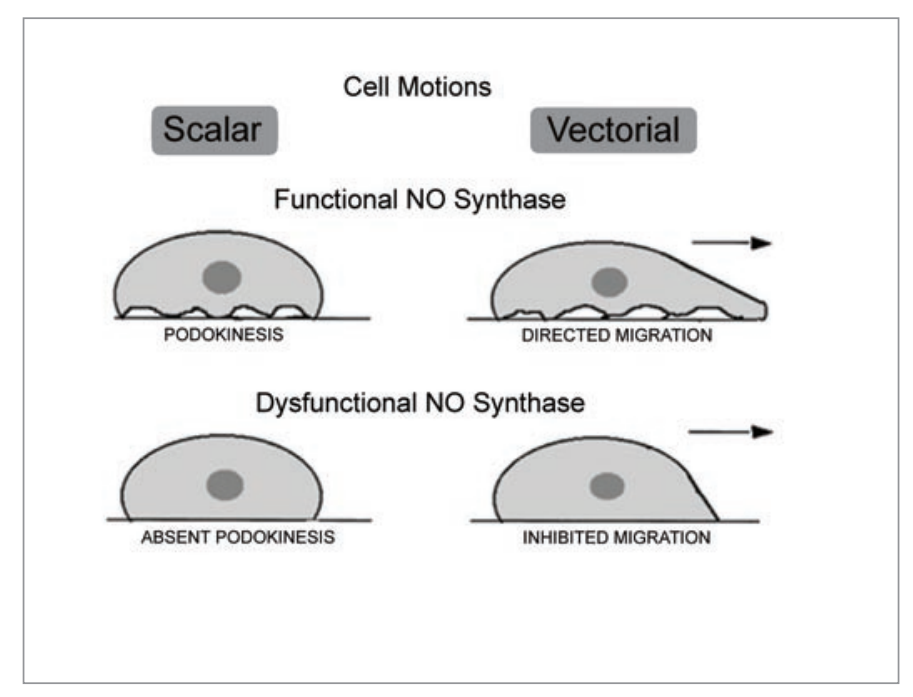

Figure 2. A schematic rendition of the concept of podokinesis-spontaneous or NO-stimulated turnover of focal adhesion complexes resulting in changes of tractional forces applied by the cells on the extracellular matrix. This is a scalar type of micromotion. This scalar motion is a prerequisite for acquisition of a directional movement when guidance cues are applied.

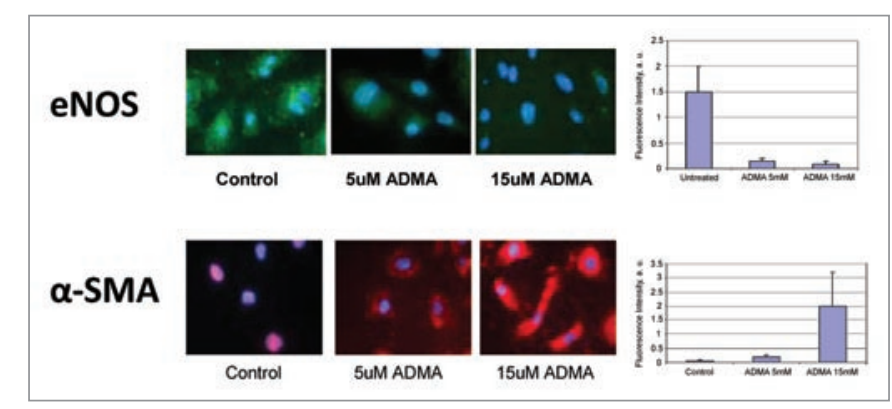

Figure 3. Cultured endothelial cells undergo endothelial mesenchymal transition induced by ADMA-loss of eNOS and acquisition of alpha-smooth muscle actin. Modified from ref. 70.

production of its anti-angiogenic fragment, endostatin lend additional support to the notion of premier role played by the renal microvasculature in the progression of disease. Indeed, our data obtained in cultured microvascular endothelial cells (Fig. 3) and in kidneys of mice chronically treated with sub-pressor doses of L-NMMA further this line of evidence. ${ }^{74}$ Uncoupling of NO synthase by ADMA or L-NMMA triggers downstream production of endostatin and TGFbeta (possibly also angiostatin), which reprogram endothelial cells to acquire myofibroblastic phenotype and initiate fibrogenic programs, as schematically depicted in Figure 4. More recently, Kalluri and co-workers have demonstrated endothelial-mesenchymal transition in a series of elegant studies of myocardial fibrosis. ${ }^{75}$

\section{Mesenchymal-Endothelial Transition}

Another poorly explored area in the field of angiogenesis is represented by the reprogramming of mesenchymal stem cells: mesenchymal-endothelial transition. Mesenchymal stem cells are resident to many if not all organs ${ }^{76}$ where they remain "sleepers" until stimulation, when cells may participate in angiogenesis, vasculogenesis and repair processes, ${ }^{77}$ as schematically illustrated in Figure 5. Angiogenic capacity of mesenchymal stem cells is based on the production of VEGF and chemoattraction of the host microvasculature, as we demonstrated using implanted angioreactors containing mesenchymal stem cells, VEGF, and FGF encapsulated into growth factor-deprived 


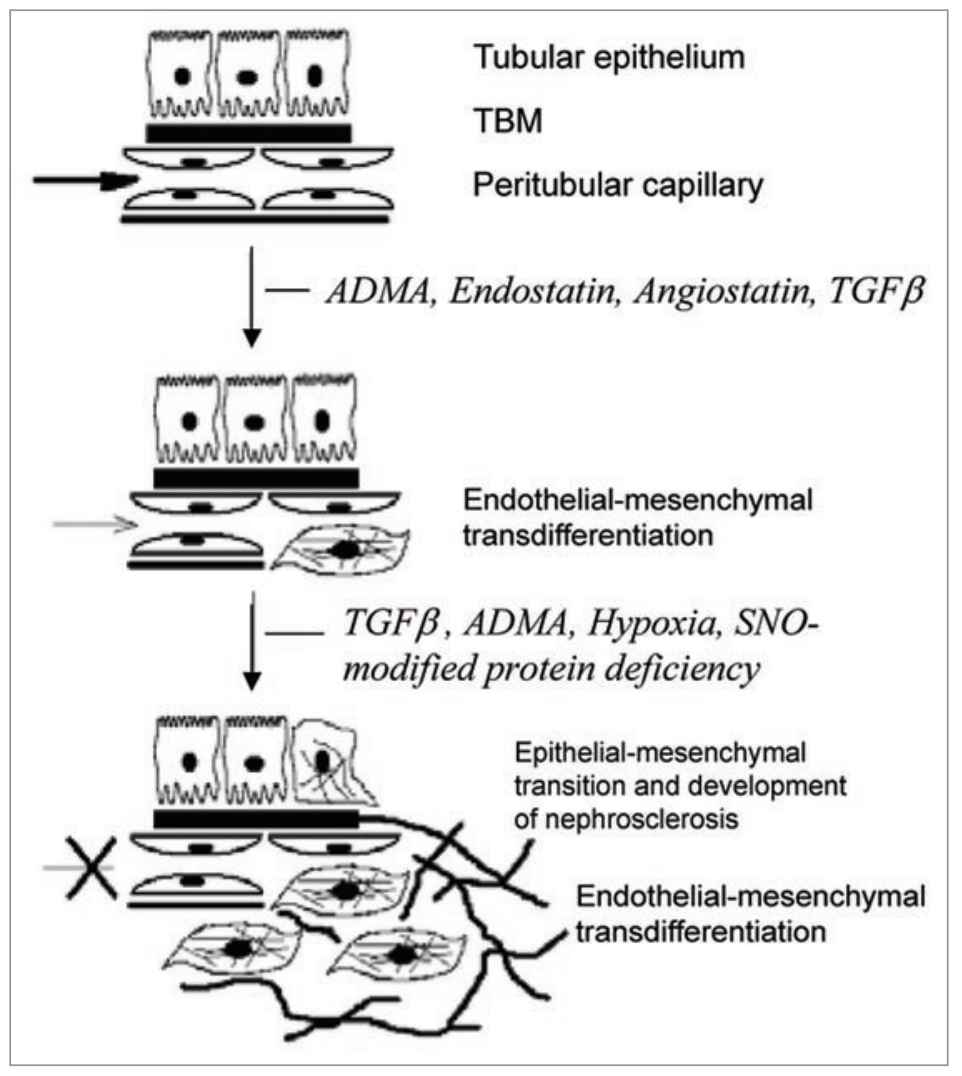

Figure 4. A hypothetical schema depicting the contribution of elevated guanidino compound, asymmetric dimethylarginine (ADMA), and downstream production of endostatin and TGFbeta to endothelial-mesenchymal transition, loss of vessel patency and fibrogenic transformation.

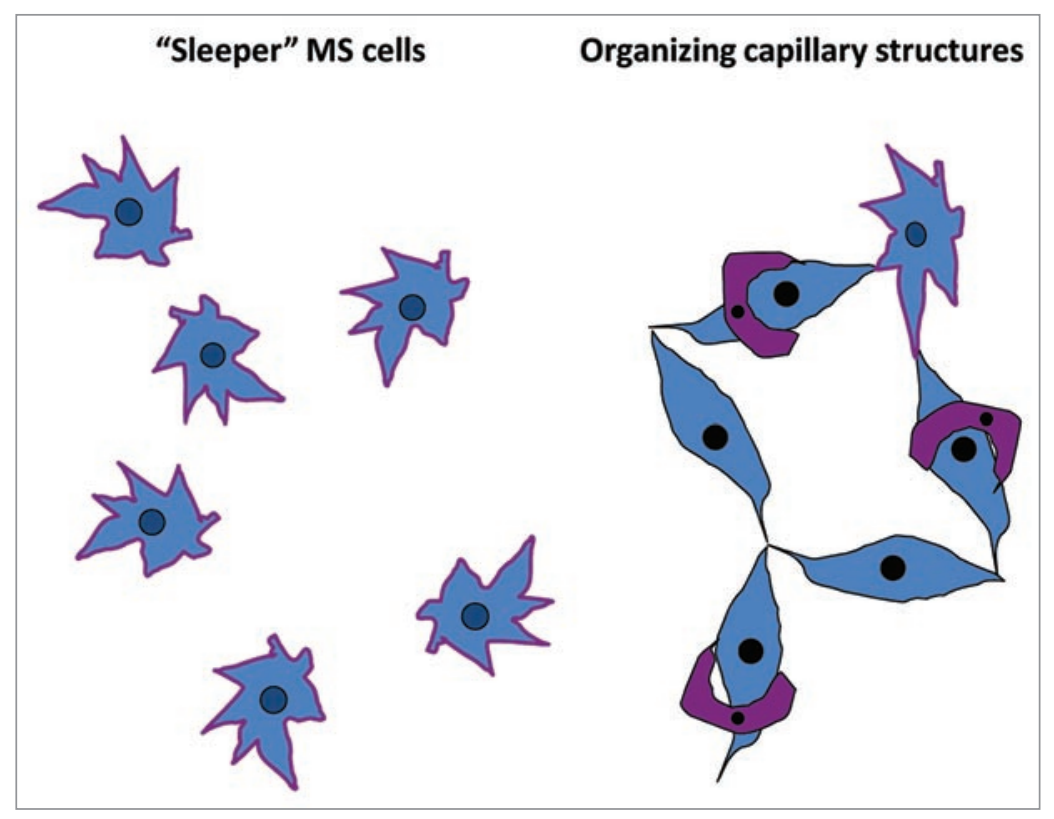

Figure 5. Mesenchymal stem cells are resident to many organs where they remain "sleepers" until stimulation, when cells may participate in angiogenesis, vasculogenesis and repair processes. matrigel. ${ }^{77}$ Having established a clonal cell line of mesenchymal stem cells obtained from kidneys of Tie-2/GFP mice, ${ }^{78}$ we sought to examine their ability to transform into endothelial cells. Under permissive conditions (high VEGF and FGF) about $60 \%$ of these cells gradually express GFP and incorporate into microvasculature. These data confirmed the ability of mesenchymal stem cells to contribute to vasculogenesis. When these cells are adoptively transferred to mice with acute renal ischemia, majority of cells (approximately $60 \%)$ express alpha-SM actin, whereas only $10 \%$ acquire Tie-2/GFP expression. Most probably, ischemic microenvironment offers non-permissive conditions for mesenchymal-endothelial transition, while it is supportive of their transformation into myofibroblasts and development of a pro-fibrotic phenotype. Learning how to regulate mesenchymal-endothelial transition may have important implications for stimulating neovascularization necessary for regenerative processes in injured organs and for inhibiting tumor angiogenesis.

\section{Chronic Allograft Nephropathy and Endorepellin}

There is growing consensus that graft vascular disease (GVD, aka allograft arteriopathy, transplant vasculopathy, graft arterial disease) is an important contributor to developing chronic allograft cardiomyopathy and nephropathy ${ }^{79}$ and can be diagnosed in about $50 \%$ patients already within the first year post-transplant. Endothelial dysfunction is its predictor and companion. ${ }^{80}$ The mechanisms of dysfunction, however, remain obscure. In this vein, our proteomic screen of patients with stable renal allograft function vs. those with a biopsy-confirmed chronic allograft nephropathy (CAN) revealed an increased excretion of a C-terminal fragment of perlecan, endorepellin, in the latter group.

Perlecan stabilizes the supramolecular structure of basement membranes partly via domain $\mathrm{V}$ binding to fibulin-2 and laminin-entactin-nidogen complex. This component also provides cross-linking to the fibrillar components and serves as a depot for growth factors like basic FGF. 


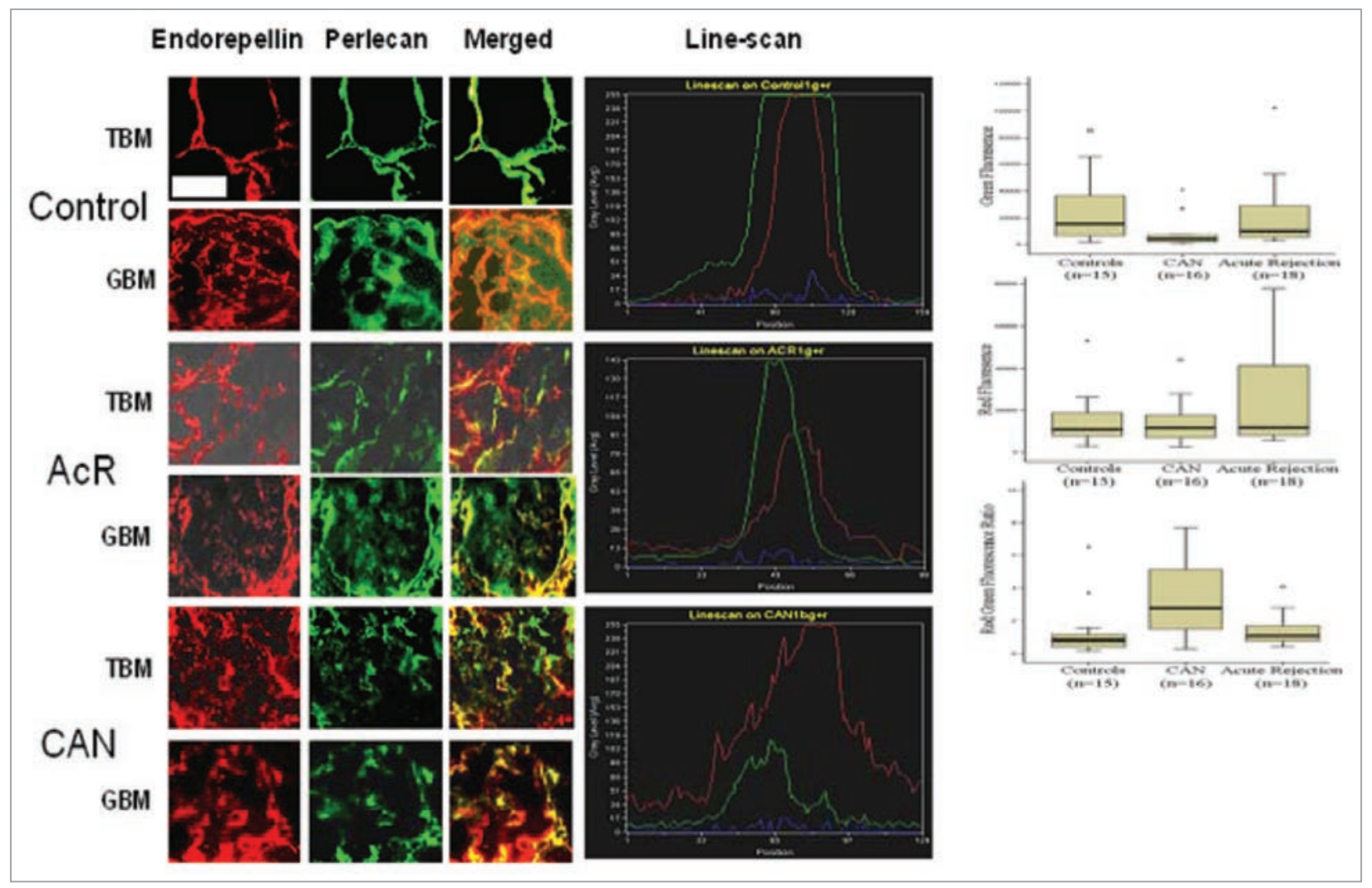

Figure 6. In patients with chronic allograft nephropathy, elevated excretion of endorepellin in the urine occurs at the expense of its parent molecule, perlecan, depletion of the latter from the glomerular and tubular basement membranes and diffusion of endorepellin into the renal parenchyma and the urine. Modified from ref. 86.

Perlecan is secreted by endothelial cells, is expressed in all basement membranes, and its deficiency in mice results in lethality on embryonic day 10.5 , when vasculogenesis commences. ${ }^{81}$ In transgenic mice with heparan sulfate-deficient perlecan, smooth muscle cells showed enhanced proliferation in vitro and intimal proliferation in vivo. ${ }^{82}$ Perlecan has been found to be atheroprotective, whereas atherogenesis has been associated with perlecan deficiency. ${ }^{83}$

The domain V, C-terminal fragment of perlecan (amino acids 3787-4391) has potent anti-angiogenic properties, providing it with a potential role in the development of a relatively avascular scar tissue as part of the process leading to progressive renal failure. ${ }^{84}$ Notably, the terminal laminin-like globular domain (LG3) of $\sim 25 \mathrm{kDa}$ can be generated by a specific proteolytic cleavage performed by BMP-1/Toll-like family of metalloproteinases, ubiquitous enzymes that are involved in tissue remodeling and inflammation. Endorepellin LG3 is a compact globular protein with a highly negative charge (pI 5), ${ }^{41}$ thus LG3 could penetrate the anionic heparan sulfates of the glomerular basement membrane. ${ }^{85}$ An identical protein fragment of endorepellin, containing amino acids 4197 to 4216 of perlecan, was found to be elevated in the urine of patients with end-stage renal disease on dialysis with concentrations estimated at $\sim 11 \mathrm{mg} / \mathrm{L} .{ }^{86}$ Endorepellin has been shown to disrupt actin stress fibers and focal adhesions via interaction with $\alpha 2 \beta 1$ integrins, thus leading to capillary pruning. ${ }^{87}$ Most recently, chronic treatment of mice bearing tumors, like human squamous cell carcinoma, with recombinant endorepellin has been shown to reduce tumor vascularization and shrink tumors. ${ }^{88}$

Appearance of endorepellin in the urine of patients with CAN occurred at the expense of the parent molecule, perlecan, as demonstrated by the results of immunohistochemical analysis and ELISA (Fig. 6). The demonstrated loss of perlecan may contribute to the development of the vasculopathy central to CAN, and the ensuing imbalance of perlecan:endorepellin may result in increased capillary pruning with subsequent scar formation. ${ }^{89}$

In conclusion, four vignettes presented herein tend to favor specialization of antiangiogenic programs over "drive-in-reverse" angiogenesis. They illustrate various routes of microvascular rarefaction taking place under diverse pathological conditions. The fact that some of the findings are based on the unbiased gene and protein microarray screening further enhances the pathogenic validity of these antiangiogenic programs by stressing their commanding presence in many of these processes.

\section{Questions and Answers}

Eduardo Slatopolsky MD, Joseph Friedman Professor of Medicine, Washington University School of Medicine: Do you think that oxidative stress plays an important role in the changes that you are observing in the endothelial-mesenchymal transition? 
Michael Goligorsky MD PhD, Alvin I Goodman Chair and Director of Renal Research Institute, New York Medical College: That is correct. When we performed similar experiments in the endothelial or neuronal nitric oxide knockout mice, the end result was quite different: there was no downregulation of aconitase- $2 .{ }^{90}$ Therefore, we came to a conclusion that, perhaps, it is uncoupled nitric oxide synthase rather than the absence of nitric oxide synthase and nitric oxide that is contributing to this phenotype. Therefore we performed experiments with Tempol: we included this antioxidant into the reaction chamber for measuring vascular tension and were able to correct acetylcholine-induced vasorelaxation.

Dr. Slatopolsky: Did you measure NADPH?

Dr. Goligorsky: No, we didn't.

Dr. Slatopolsky: Would expect it to increase?

Dr. Goligorsky: We'll have to measure it to answer your question.

Scott Boyle PhD, Postdoctoral fellow in nephrology, Washington University School of Medicine: Was the cell line from the Tie2/GFP mice derived from embryonic kidneys or was the method designed to isolate a mesenchymal population from an adult kidney? Did you see the cells turn into anything else besides endothelium or smooth muscle cells either in vivo or in vitro?

Dr. Goligorsky: Dr. Plotkin in our Division has isolated these cells from collagenase-dispersed kidneys of adult Tie-2/ GFP mice and, using traditional plastic adhesion techniques, enriched mesenchymal stem cells. ${ }^{78} \mathrm{He}$ obtained and studied several clones and demonstrated that they can be reprogrammed toward osteoblastic, chondrocytic, adipocytic phenotype, as well as become smooth muscle and endothelial cells.

Kyunghee Choi PhD, Associate Professor of Pathology and Immunology, Washington University School of Medicine: Just a follow up on that question. I believe you said that while cultured cells can undergo transition, the freshly isolated cells cannot. Do you think the data may reflect an artifact of culturing? Also, how do you know that what you judge to be transition is not cell fusion?
Dr. Goligorsky: Yes, this is a cloned cell line and Dr. Plotkin ${ }^{78}$ has characterized them exhaustively. I agree with you, we don't have clear cut evidence that there were no fusion events. These cells express Tie2/GFP, which is a marker of endothelial cells, but I agree with you, it may be expressed in other cells as well. The fact that these cells are incorporated into the vasculature provides additional support. ${ }^{77}$ But, I agree it would be imperative to demonstrate that they do express some additional endothelial functions, like nitric oxide production, antithrombogenic properties, etc. We haven't done it. I agree with you, this is a gap that remains to be filled.

Feng Chen PhD, Assistant Professor of Medicine, Washington University School of Medicine: Do you see any similarity between the pathological endothelial-to-mesenchymal transition you describe and the endothelial to mesenchymal transition that occurs normally during embryonic development? For example, the formation of heart valves requires endothelial or endocardial to mesenchymal transition. Have you looked at some of the key genes involved in this process, such as NFATc1 and Sox9?

Dr. Goligorsky: You are absolutely right. During embryogenesis, myocardial cushion undergoes endothelial-mesenchymal transformation (reviewed in ref. 73). There was a demonstration that endothelial mesenchymal transformation might occur in adult animals after exposure to $\mathrm{x}$-ray irradiation. Dr. O'Riordan in our laboratory provided in vitro and in vivo data related to a blockade of nitric oxide synthase using sub-pressor doses of a nitric oxide synthase inhibitor. ${ }^{73}$ More recently Dr. Kalluri also confirmed the possibility of endothelial-mesenchymal transition in the case of cardiomyopathy. ${ }^{75} \mathrm{I}$ am not aware of studies examining the role of NFATc1 or Sox9 in this process.

Maggie Chen, MD PhD, Postdoctoral fellow in nephrology, Washington University School of Medicine: In your experiments, you showed VEGF-induced mesenchymal-to-endothelial transition. Have you examined whether angiopoetin-1 or angiopoetin-2 also promotes the transition?
Dr. Goligorsky: No we haven't. In the studies presented we used VEGFcontaining endothelial specific culture medium to maintain mesenchymal stem cells for 3-4 weeks until Tie-2 promotor-driven GFP become detectable. ${ }^{77} \mathrm{We}$ haven't come to check effects of angiopoietins yet.

\section{Acknowledgements}

Dr. Goligorsky's studies were supported in part by NIH grants DK54602, DK052783 and DK45462 and Westchester Artificial Kidney Foundation. Organogenesis Forum Conferences are supported by the Washington University George M. O’Brien Center DK079333 and participated in by Washington University Faculty.

\section{References}

1. Folkman J. Tumor angiogenesis. Adv Cancer Res 1985; 43:175-203.

2. Carmeliet P. Mechanisms of angiogenesis and arteriogenesis. Nat Med 2000; 6:389-95.

3. Dvorak H. How tumors make bad blood vessels and stroma. Am J Pathol 2003; 162:1747-57.

4. Yancopoulos G, Davis S, Gale N, Rudge J, Wiegand $\mathrm{S}$, Holash J. Vascular-specific growth factors and blood vessel formation. Nat 2000; 407:242-8.

5. Carmeliet P, Jain R. Angiogenesis in cancer and other diseases. Nat 2000; 407:249-57.

6. Augustin H, Koh G, Thurston G, Alitalo K. Control of vascular morphogenesis and homeostasis through the angiopoietin-Tie system. Nat Rev Mol Cell Biol 2009; 10:165-77.

7. Benest A, Augustin H. Tension in the vasculature Nat Med 2009; 15:608-10.

8. Folkow B. The Forth Volhard lecture: cardiovascular structural adaptation: its role in the initiation and maintenance of primary hypertension. Clin Sci Mol Med 1978; 4:3-22.

9. Prewitt R, Chen I, Dowell R. Development of microvascular rarefaction in the spontaneously hypertensive rat. Am J Physiol 1982; 243:243-51.

10. Prewitt R, Cardoso S, Wood W. Prevention of arteriolar rarefaction in the spontaneously hypertensive rat by exposure to simulated high altitude. J Hypertens 1986; 4:735-40.

11. Hashimoto H, Prewitt R, Efaw C. Alterations in the microvasculature of one-kidney, one-clip hypertensive rats. Am J Physiol 1987; 253:933-40.

12. Boegenhold M, Johnson M, Overbeck H. Pressureindependent arteriolar rarefaction in hypertension. Am J Physiol 1991; 261:83-7.

13. Hansen-Smith F, Morris L, Green A, Lombard J. Rapid microvessel rarefaction with elevated salt intake and reduced renal mass. Circ Res 1996; 79:324-30.

14. Rieder M, Roman R, Greene A. Hypertension 1997; 30:120-7.

15. Papanek P, Rieder M, Lombard J, Greene A. Genderspecific protection from microvessel rarefaction in female hypertensive rats. Am J Hypertens 1998; 11:998-1005

16. Gobe G, Browning J, Howard T, Hogg N, Winterford C, Cross R. Apoptosis occurs in endothelial cells during hypertension-induced microvascular rarefaction. J Struct Biol 1997; 118:63-72. 
17. Nusz D, White D, Dai Q, Pippen A, Thompson M, Walton G, et al. Vasular rarefaction in peripheral skeletal muscle after experimental heart failure. Am J Physiol 2003; 285:1554-62.

18. Sladek T, Gerova M, Znojil V, Devat L. Morphometric characteristics of cardiac hypertrophy induced by long-term inhibition of NO synthase. Physiol Res 1996; 45:335-8.

19. Kubis N, Richer C, Domergue V, Giudicelli J, Levy B. Role of microvascular rarefaction in the increased arterial pressure in mice lacking the endothelial nitric oxide synthase gene. J Hypertens 2002; 20:1581-7.

20. Okruhlicova L, Tribulova N, Weismann P, Sotnikova R. Ultrastructure and histochemistry of rat myocardial capillary endothelial cells in response to diabetes and hypertension. Cell Res 2005; 15:532-8.

21. Tornig J, Amann K, Ritz E, Nichols C, Zeier M, Mall G. Arteriolar thickening, capillary rarefaction and interstitial fibrosis in the heart of rats with renal failure: the effects of ramipril, nifedipine and moxonidine. J Am Soc Nephrol 1996; 7:667-75.

22. Sabri A, Samuel J, Marotte F, Poitevin P, Pappaport L, Levy B. Microvasculature in angiotensin II-dependent cardiac hypertrophy in the rat. Hypertension 1998; 32:371-5.

23. Sokolova I, Manukhina E, Blinkov S, Koshelev V, Pinelis V, Rodionov I. Rarefaction of arterioles and capillary network in the brain of rats with different forms of hypertension. Microvasc Res 1985; 30:1-9.

24. Sonntag W, Lynch C, Cooney P, Hutchins P. Decreases in cerebral microvasculature with age are associated with the decline in growth hormone and insulin-like growth factor-1. Endocrinol 1997; 138:3515-20.

25. Harper R, Moore M, Marr M, Watts L, Hutchins P. Arteriolar rarefaction in the conjunctiva of human essential hypertension. Microvasc Res 1978; 16:36972.

26. Funk R, Rohen J. Comparative morphological studies on blood vessels in eyes of normotensive and spontaneously hypertensive rats. Exp Eye Res 1985; 40:191-203.

27. Keshet E. Preventing pathological regression of blood vessels. J Clin Invest 2003; 112:27-9.

28. Montagna W, Carlisle K. Structural changes in aging human skin. J Invest Dermatol 1979; 73:47-53.

29. Hasan K, Manyonda I, Ng F, Singer D, Antonios T. Skin capillary density changes in normal pregnancy and preeclampsia. J Hypertens 2002; 20:2439-43.

30. Houben A, Beljaars J, Hofstra L, Kroon A, De Leeuw P. Microvascular abnormalities in chronic heart failure: a cross-sectional study. Microcirculation 2003; 10:471-8.

31. Serne E, Gans R, ter Maaten J, Tangelder G, Donker A, Stehouwer C. Impaired skin capillary recruitment in essential hypertension is caused by both functional and structural capillary rarefaction. Hypertension 2001; 38:238-42.

32. Stewart J, Kohen A, Brouder D, Rahim F, Adler S, Garrick R, Goligorsky MS. Non-invasive interrogation of microvasculature for sigfns of endothelial dysfunction in patients with chronic renal failure. Am J Physiol Heart 2004; 287:2687-96.

33. Bohle A, Mackensen-Haen S, von Gise H. Significance of tubulointerstitial changes in the renal cortex for the excretory function and concentration ability of the kidney. Am J Nephrol 1987; 7:421-33.

34. Lee L, Meyer T, Pollock A, Lovett D. Endothelial cell injury initiates glomerular sclerosis in the rat remnant kidney. J Clin Invest 1995; 96:953-64.

35. Kang D, Joly A, Mazali M, Johnson R. Impaired angiogenesis in the remnant kidney model. J Am Soc Nephrol 2001; 12:1448-57.

36. Kang D, Nakagawa T, Feng L, Johnson R. Nitric oxide modulates vascular disease in the remnant kidney model. Am J Pathol 2002; 161:239-48.
37. Kang D, Kanellis J, Hugo C, Johnson R. Role of microvascular endothelium in progressive renal disease. J Am Soc Nephrol 2002; 13:806-16.

38. Ohashi R, Kitamura H, Yamanaka N, Johnson R. Peritubular capillary injury during progression of experimental glomerulonephritis in rats. J Am Soc Nephrol 2000; 11:47-56.

39. Ohashi R, Shimizu A, Masuda Y, Johnson R. Peritubular capillary regression during progression of experimental obstructive nephropathy. J Am Soc Nephrol 2002; 13:1795-805.

40. Gealikman O, Brodsky SV, Zhang F, Chander P, Friedli C, Nasjletti A, Goligorsky MS. Angiogenic incompetence and microvasculopathy in the Zucke diabetic fat rat are ameliorated with Ebselen treatment: endothelial dysfunction as a modifier of angiogenic response. Kidney Int 2004; 66:2337-47.

41. Namikoshi T, Satoh M, Horike H, Fujimoto $S$, Arakawa S, Sasaki T, Kashihara N. Implications of peritubular capillary loss and altered expression of vascular endothelial growth factor in IgA nephropathy. Nephron 2006; 102:9-16

42. Vollmar B, Siegmund S, Richter S, Menger M Microvascular consequences of Kuppfer cell modulation in rat liver fibrogenesis. J Pathol 1999; 189:8591.

43. Hudetz A. Percolation phenomenon: the effect of capillary network rarefaction. Microvasc Res 1993; 45:1-10.

44. Greene A, Tonelato P, Lui J, Lombard J, Cowley A. Microvascular rarefaction and tissue vascular resis tance in hypertension. Am J Physiol 1989; 256:12631.

45. Shih S-C, Ju M, Liu N, Smith L. Selective stimulation of VEGFR1 prevents oxygen-induced retinal vascular degeneration in retinopathy of prematurity. J Clin Invest 2003; 112:50-7.

46. Carmeliet P. Angiogenesis in health and disease. Nat Med 2003; 9:653-60

47. O'Reilly M, Boehm T, Shing Y, Fukai N, Vasios G, Lane W, et al. Endostatin: an endogenous inhibitor of angiogenesis and tumor growth. Cell 1997; 88:27785.

48. Folkman J. Antiangiogenesis in cancer therapyendostatin and its mechanism of action. Exp Cell Res 2006; 312:594-607

49. Im E, Venkatakrishnan A, Kazlauskas A. Cathepsin $B$ regulates the intrinsic angiogenic threshold of endothelial cells. Mol Biol Cell 2005; 16:3488-500.

50. Rehn M, Veikkola T, Kukk-Valdre E, Nakamura $\mathrm{H}$, Itmonen $\mathrm{M}$, Lombardo $\mathrm{C}$, et al. Interaction of endostatin with integrins implicated in angiogenesis. Proc Natl Acad Sci USA 2001; 98:1024-9.

51. Bates D, Cui T, Doughty J, Winkler M, Sugino M, Shields J, et al. VEGF165b, an inhibitory splice variant of VEGF, is downregulated in renal cell carcinoma. Cancer Res 2002; 62:4123-31.

52. Perrin R, Konopatskaya O, Qiu Y, Harper S, Bate D, Churchill A. Diabetic retinopathy is associated with a switch in splicing from anti- to pro-angiogenic isoforms of VEGF. Diabetologia 2005; 48:2422-7.

53. Noiri E, Peresleni T, Srivastava N, Weber P, Bahou W, Peunova N, Goligorsky MS. Nitric oxide is necessary for a switch from stationary to locomoting phenotype in epithelial cells. Am J Physiology 1996; 270:794-802.

54. Noiri E, Hu Y, Bahou WF, Keese C, Giaever I, Goligorsky MS. Permissive role of nitric oxide in endothelin-induced migration of endothelial cells. J Biol Chem 1997; 272:1747-52.

55. Noiri E, Lee E, Testa J, Quigley J, Colflesh D, Keese $\mathrm{C}$, et al. Podokinesis in endothelial cell migration role of NO. Am J Physiol Cell 1998; 274:236-44.

56. Yung Y, Chae J, Buehler M, Hunter C, Mooney D. Cyclic tensile strain triggers a sequence of autocrine and paracrine signaling to regulate angiogenic sprouting in human vascular cells. Proc Natl Acad Sci USA 2009 ; 106:15279-84.
57. Kilarski W, Samolov B, Petersson L, Kvanta A Gerwins P. Biomechanical regulation of blood vessel growth during tissue vascularization. Nat Med 2009; 15:657-64.

58. Ausprunk D, Falterman K, Folkman J. The sequence of events in the regression of corneal capillaries. Lab Invest 1978; 38:284-94.

59. Ito M, Yoshioka M. Regression of the hyaloids vessels and papillary membrane of the mouse. Anat Embryol (Berl) 1999; 200:403-11.

60. Lobov I, Rao S, Carroll T, Vallance J, Ito M, Ondr $\mathrm{J}$, et al. WNT7b mediates macrophage-induced programmed cell death in patterning of the vasculature. Nat 2005; 437:417-21.

61. Jurasz P, Alonso D, Castro-Blanco S, Murad F, Radomski M. Generation and role of angiostatin in human platelets. Blood 2003; 102:3217-23.

62. VonTell D, Armulik A, Betscholtz C. Pericytes and vascular stability. Exp Cell Res 2006; 312:623-9.

63. Yemisci M, Gursoy-Ozdemir Y, Vural A, Can A, Topalkara K, Dalkara T. Pericyte contraction induced by oxidative-nitrosative stress impairs capillary reflow despite successful opening of an occluded cerebral artery. Nat Med 2009; 15:1031-8.

64. Yamashita J, Itoh $\mathrm{H}$, Hirashima M, Ogawa M, Nishikawa S, Yurugi T, et al. Flk-1-positive cells derived from embryonic stem cells serve as vascular progenitors. Nature 2000; 408:92-6.

65. DeRuiter M, Poelmann R, VanMunsteren J, Mironov V, Markwald R, Gittenberger-de Groot A. Embryonic endothelial cells transdifferentiate into mesenchymal cells expressing smooth muscle actins in vivo and in vitro. Circ Res 1997; 80:444-51.

66. Frid M, Kale V, Stenmark K. Mature vascular endothelium can give rise to smooth muscle cells via endothelial-mesenchymal transdifferentiation. Circ Res 2002; 90:1189-96.

67. Ishizaki A, Hayashi H, Li A, Imamura T. Human umbilical vein endothelium-derived cells retain potential to differentiate into smooth muscle-like cells. J Biol Chem 2003; 278:1303-9.

68. Hillebrands J, Klatter F, van den Hurk B, Popa E, Nieuwenhuis P, Rozing J. Origin of neointima endothelium and alpha-actin-positive smooth muscle cells in transplant arteriosclerosis. J Clin Invest 2001; 107:1411-22

69. Iwano M, Plieth D, Danoff T, Xue C, Okada $H$, Neilson E. Evidence that fibroblasts derive from epithelium during tissue fibrosis. J Clin Invest 2002; 110:341-50.

70. Kalluri R, Neilson E. Epithelial-mesenchymal transition and its implications for fibrosis. J Clin Invest 2003; 112:1776-84

71. Thiery JP. Epithelial-mesenchymal transitions in development and pathologies. Curr Opinion Cell Biol 2003; 15:740-6.

72. Liu J. Epithelial to mesenchymal transition in renal fibrogenesis: pathologic significance, molecular mechanisms and therapeutic intervention. J Am Soc Nephrol 2004; 15:1-12.

73. O'Riordan E, Mendelev N, Patschan S, Patschan D, Eskander J, Cohen-Gould L, et al. Chronic NOS inhibition actuates endothelial-mesenchymal transformation. Am J Physiol Heart Circ Physiol 2007; 292:285-94

74. Stossel A, Paliege A, Thelig F, Addabbo F, Patschan D, Goligorsky MS, Bachmann S. Indolent course of tubulointerstitial disease in a mouse model of subpressor, low-dose nitric oxide synthase inhibition. Am J Physiol Renal 2008; 295:717-25.

75. Zeisberg EM, Tarnavski O, Zeisberg M, Dorfman AL, McMullen JR, Gustafsson E, et al. Endothelialto-mesenchymal transition contributes to cardiac fibrosis. Nat Med 2007; 13:952-61.

76. da Silva Meirelles L, Chagastelles PC, Nardi NB. Mesenchymal stem cells reside in virtually all postnatal organs and tissues. J Cell Sci 2006; 119:220413. 
77. Chen J, Park H-C, Pelger E, Li H, Plotkin M, Goligorsky MS. Mesenchymal stem cells participate in vasculogenesis, angiogenesis and endothelial repair. Kidney Interntl 2008; 74:879-89.

78. Plotkin MD, Goligorsky MS. Mesenchymal cells from adult kidney support angiogenesis and differentiate into multiple interstitial cell types including erythropoietin-producing fibroblasts. Am J Physiol Renal Physiol 2006; 291:902-12.

79. Mitchell R. Graft vascular disease: immune response meets vascular wall. Annu Rev Pathol Mech Dis 2009; 4:19-47.

80. Davis S, Yeung A, Meredith I, Charbonneau F, Ganz P. Early endothelial dysfunction predicts the development of transplant coronary artery disease at 1 year posttransplant. Circ 1996; 93:457-62.

81. Iozzo RV. Matrix proteoglycans: from molecular design to cellular function. Annu Rev Biochem 1998; 67:609-52.
82. Tran PK, Tran-Lundmark K, Soininen R, Tryggvason $\mathrm{K}$, Thyberg J, Hedin U. Increased intimal hyperplasia and smooth muscle cell proliferation in transgenic mice with heparan sulfate-deficient perlecan. Circ Res 2004; 94:550-8.

83. Pillarisetti S. Lipoprotein modulation of subendothelial heparan sulfate proteoglycans (perlecan) and atherogenicity. Trends Cardiovasc Med 2000; 10:60 5.

84. Mongiat M, Sweeney SM, San Antonio JD, Fu J, Iozzo RV. Endorepellin, a novel inhibitor of angiogenesis derived from the $\mathrm{C}$ terminus of perlecan. J Biol Chem 2003; 278:4238-49.

85. Gonzalez EM, Reed CC, Bix G, Fu J, Zhang Y, Gopalakrishnan B, et al. BMP-1/Tolloid-like metalloproteases process endorepellin, the angiostatic C-terminal fragment of perlecan. J Biol Chem 2005; 280:7080-7.

86. Oda O, Shinzato T, Ohbayashi K, Takai I, Kunimatsu $\mathrm{M}$, Maeda K, et al. Purification and characterization of perlecan fragment in urine of end-stage renal failure patients. Clin Chim Acta 1996; 255:119-32.
87. Bix G, Fu J, Gonzalez EM, Macro L, Barker A, Campbell S, et al. Endorepellin causes endothelial cell disassembly of actin cytoskeleton and focal adhesions through alpha2beta1 integrin. J Cell Biol 2004; 166:97-109.

88. Bix G, Castello R, Burrows M, Zoeller J, Weech M, Iozzo R, et al. Endorepellin in vivo: targeting tumor vasculature and retarding cancer growth and metabolism. J Natl Cancer Inst 2006; 98:1634-46.

89. O'Riordan E, Orlova TN, Mendelev N, Patschan D, Kemp R, Chander PN, et al. Urinary proteomic analysis of chronic allograft nephropathy. ProteomicsClinical Applications 2008; 2:1025-35.

90. Addabbo F, Ratliff B, Park H-C, Kuo MC, Sodhi K, Zhang F, et al. Mitochondrial function and mass are early victims of endothelial dysfunction. Am J Pathol 2009; 174:34-43. 